\title{
Capture of the Late Phase of Long-Term Potentiation within and across the Apical and Basilar Dendritic Compartments of CA1 Pyramidal Neurons: Synaptic Tagging Is Compartment Restricted
}

\author{
Juan M. Alarcon, ${ }^{1,2}$ Angel Barco, ${ }^{1,4}$ and Eric R. Kandel ${ }^{1,2,3}$ \\ ${ }^{1}$ Center for Neurobiology and Behavior, ${ }^{2}$ Kavli Institute for Brain Sciences, and ${ }^{3}$ Howard Hughes Medical Institute, College of Physicians and Surgeons of \\ Columbia University, New York, New York 10032, and ${ }^{4}$ Instituto de Neurociencias de Alicante, Universidad Miguel Hernandez, San Juan de Alicante 03550, \\ Spain
}

\begin{abstract}
Studies in the rodent hippocampus have demonstrated that when the late phase of long-term potentiation (L-LTP) is induced in a set of synapses by suprathreshold stimulation, L-LTP can also be expressed by other synapses receiving subthreshold stimulation, a phenomenon usually referred as "capture of L-LTP." Because the pyramidal neurons in the mammalian hippocampus have both apical and basal dendrites, we have now investigated whether capture of L-LTP, previously described only within the apical dendritic compartment, can also take place within the basilar dendritic compartment and, if so, whether capture can be accomplished from one dendritic compartment to the other. We found that capture of L-LTP can also occur within the basilar dendritic compartment and that the tagging signal that enables capture appears to be the same in both dendritic compartments. However, capture across compartments, between the apical and basilar dendrites, follows different rules and requires a stronger triggering stimulation than capture within a compartment. These results suggest that the tag appears specific to a compartment either apical or basilar and that an additional mechanism may be required to capture across compartments.
\end{abstract}

Key words: hippocampus; synaptic capture; synaptic tagging; long-term potentiation; dendritic compartment; dendritic tagging

\section{Introduction}

The induction and maintenance of the late phase of long-term potentiation (L-LTP) require the activation of a molecular cascade that includes signaling to the nucleus, alterations in gene expression, and the synthesis of new gene products that stabilize LTP in activated synapses (Kandel, 2001). Different models have been proposed to explain how new gene products come to reside into activated synapses. In the "mail" hypothesis, the newly synthesized mRNAs and proteins are sent specifically to activated synapses. In the "synaptic tag" model, the new gene products reach all of the synapses, both active and inactive, but those gene products can only be captured and used productively at those synapses that have been tagged by local activity, a phenomenon called "synaptic tagging" (Frey and Morris, 1997). The synaptic tag model has been supported by a number of studies in the rodent hippocampus (Frey and Morris, 1998a,b; Barco et al., 2002; Martin and Kosik, 2002;

Received Aug. 1, 2005; revised 0ct. 11, 2005; accepted Nov. 9, 2005.

E.R.K. is supported by the Howard Hughes Medical Institute. We thank Hsiu-Ling Li, Isabel Muzzio, Russel Nicholls, Mathew Nolan, Steven Siegelbaum, Martin Theis, and Sebastian Thuault for critical reading of this manuscript and all of the members of Kandel laboratory for helpful comments.

Correspondence should be addressed to Eric R. Kandel, Center for Neurobiology and Behavior, College of Physicians and Surgeons, Columbia University, 1051 Riverside Drive, New York, NY 10032. E-mail: erk5@columbia.edu. DOI:10.1523/JNEUROSCI.3196-05.2006

Copyright $\odot 2006$ Society for Neuroscience $\quad$ 0270-6474/06/260256-09\$15.00/0
Pang and Lu, 2004; Barco et al., 2005) and the sea snail Aplysia (Martin et al., 1997; Casadio et al., 1999; Martin, 2002).

The studies in the hippocampus have been limited to examining tagging and capture within a dendritic compartment (Frey and Morris, 1997, 1998a; Barco et al., 2002). However, a typical CA1 pyramidal neuron has both an apical and a basilar compartment that are distinctively innervated by other CA1 neurons, CA3 neurons, the entorhinal cortex, the amygdaloid complex, and the thalamus (Amaral and Witter, 1989; Deuchars and Thomson, 1996; Dolleman-Van Der Weel and Witter, 1996; Pikkarainen et al., 1999). The information received from these different inputs is processed and integrated in the apical and basilar dendrites of the CA1 pyramidal neuron to enable the encoding and storage of spatial, contextual, and relational information. Apical and basilar dendrites also differ in morphology, biophysical properties, and LTP induction and expression (Arai et al., 1994; Haley et al., 1996; Son et al., 1996; Cavus and Teyler, 1998; Leung and Shen, 1999; Kloosterman et al., 2001; Kawakami et al., 2003; Kramar and Lynch, 2003). These differences could conceivably play an important role during the integration of afferent inputs in CA1 pyramidal neurons.

Here we examine both the basal and the apical dendrites, and we asked two questions. (1) Does synaptic capture take place in the basilar compartment similarly to what is observed in the api- 
Table 1. Two-pathway paired-pulse facilitation between proximal (S1) and distal (S2) inputs in the stratum radiatum (mean \pm SD)

\begin{tabular}{|c|c|c|c|c|}
\hline \multirow{2}{*}{$\begin{array}{l}\text { Interpulse } \\
\text { interval (msec) }\end{array}$} & First pulse & Second pulse ${ }^{a}$ & First pulse & Second pulse ${ }^{b}$ \\
\hline & S1 & S2 & S2 & S1 \\
\hline 50 & $100 \%$ & $98 \pm 7 \%$ & $100 \%$ & $95 \pm 6 \%$ \\
\hline 100 & $100 \%$ & $99 \pm 7 \%$ & $100 \%$ & $103 \pm 7 \%$ \\
\hline
\end{tabular}

${ }^{a}$ Change (percentage) in the S2 fEPSP slope amplitude after $\mathrm{S} 1$ stimulation.

${ }^{b}$ Change (percentage) in the S1 fEPSP slope amplitude after S2 stimulation.

cal compartment? (2) Does capture occur across dendritic compartments?

We found that capture of L-LTP within the apical or basilar dendritic areas appears to require the same physiological tagging process (one train of tetanic stimulation). In contrast, the capture of L-LTP across dendritic compartments follows different rules. Cross-compartment capture could only be achieved with a stronger triggering stimulation, such as two trains of tetanic stimulation. The compartmental restriction of synaptic tagging suggests a model according to which the availability of gene products after the onset of L-LTP is not neuron wide but compartment specific. An additional and yet unspecified activity-mediated mechanism is necessary for transcompartmental capture.

\section{Materials and Methods}

Electrophysiology. Transverse hippocampal slices $(400 \mu \mathrm{m})$ from C57BL/6 mice (3-4 months old) were incubated in an interface chamber (tissue slice recording chamber 21000-03; Fine Science Tools, Foster City, CA) at $27-28^{\circ} \mathrm{C}$, subfused (flow rate of $6 \mathrm{ml} / \mathrm{min}$ ) with oxygenated artificial CSF (ACSF) (in mM: $119 \mathrm{NaCl}, 4.0 \mathrm{KCl}, 1.5$ $\mathrm{MgSO}_{4}, 2.5 \mathrm{CaCl}_{2}, 26.2 \mathrm{NaHCO}_{3}, 1 \mathrm{NaH}_{2} \mathrm{PO}_{4}$, and 11 glucose), and allowed to equilibrate for at least $120 \mathrm{~min}$. All procedures were performed in compliance with the Institutional Animal Care and Use Regulations of Columbia University. Field EPSP (fEPSPs) from CA1 pyramidal cells were recorded from either apical or basal dendritic compartments by placing both stimulating (concentric bipolar electrode CBARD60; Frederick Haer Company, Bowdoinham, ME) and recording (micropipette filled with ACSF; pipette resistance of 5-10 $\mathrm{M} \Omega$ ) electrodes in the stratum radiatum or stratum oriens of the CA1 area, respectively. Before every experiment, synaptic input/output curves were generated, and the stimulation intensity was adjusted to give fEPSP slopes of $\sim 40 \%$ of maximum. Baseline, during, and after stimuli responses were sampled once per minute at this intensity (test pulse duration of $50 \mu \mathrm{s}$ ). Dual recording within the apical dendritic compartment was performed as described previously (Barco et al., 2002). By placing one stimulating and one recording electrode in the stratum oriens and another pair of electrodes in the stratum radiatum, we performed simultaneous recording from the basilar and apical dendritic compartments. Both recording electrodes tips formed a vertical line that was perpendicular to the line formed by the stratum pyramidale. For each set of dual-recording experiments, we ensured pathway independency by testing paired-pulse facilitation (Katz and Miledi, 1970) at 50 and $100 \mathrm{~ms}$ interpulse intervals between afferents within the apical dendritic area (Table 1), within the basal dendritic compartment (Table 2), and across dendritic compartments (Table 3). For two-pathway experiments in the stratum oriens, we chose stimulating electrodes with narrower tips (concentric bipolar electrode CBAED75; Frederick Haer Company) to minimize crossinterference between pathways attributable to the spatial restriction in that dendritic compartment.

Drugs. Actinomycin-D at $40 \mu \mathrm{M}$ (ICN Biomedicals, Aurora, OH), anisomycin at $20 \mu \mathrm{M}$, and $\mathrm{KT} 5720[(9 S, 10 R, 12 R)-2,3,9,10,11,12-$ hexahydro-10-hydroxy-9-methyl-1-oxo-9,12-epoxy- $1 \mathrm{H}$-diindolo[1,2,3-fg: $3^{\prime}, 2^{\prime}, 1^{\prime}$-kl] pyrrolo[3,4-i] [1,6] benzodiazocine-10-carboxylicacid hexyl ester] at $1 \mu \mathrm{M}$ (Calbiochem, La Jolla, CA) and kynurenic acid at $3 \mathrm{~mm}$ (Sigma, St. Louis, MO) were used. When necessary, dimethylsulfoxide (DMSO) (Sigma) was used to help dissolve a drug (DMSO final
Table 2. Two-pathway paired-pulse facilitation between proximal (S2) and distal (S1) inputs in the stratum oriens (mean \pm SD)

\begin{tabular}{|c|c|c|c|c|}
\hline \multirow{2}{*}{$\begin{array}{l}\text { Interpulse } \\
\text { interval (msec) }\end{array}$} & First pulse & Second pulse $e^{a}$ & First pulse & Second pulse \\
\hline & $S 1$ & S2 & S2 & S1 \\
\hline 50 & $100 \%$ & $103 \pm 6 \%$ & $100 \%$ & $107 \pm 7 \%$ \\
\hline 100 & $100 \%$ & $105 \pm 7 \%$ & $100 \%$ & $106 \pm 8 \%$ \\
\hline
\end{tabular}

${ }^{a}$ Change (percentage) in the S2 fEPSP slope amplitude after S1 stimulation.

${ }^{b}$ Change (percentage) in the $\mathrm{S} 1 \mathrm{fEPSP}$ slope amplitude after $\$ 2$ stimulation.

Table 3. Two-pathway paired-pulse facilitation between inputs in the stratum oriens (S1) and the stratum radiatum (S2) (mean \pm SD)

\begin{tabular}{|c|c|c|c|c|}
\hline \multirow{2}{*}{$\begin{array}{l}\text { Interpulse } \\
\text { interval (msec) }\end{array}$} & First pulse & Second pulse $e^{a}$ & First pulse & Second pulse \\
\hline & S1 & S2 & S2 & S1 \\
\hline 50 & $100 \%$ & $99 \pm 6 \%$ & $100 \%$ & $98 \pm 9 \%$ \\
\hline 100 & $100 \%$ & $99 \pm 5 \%$ & $100 \%$ & $98 \pm 12 \%$ \\
\hline
\end{tabular}

${ }^{a}$ Change (percentage) in the S2 fEPSP slope amplitude after $\mathrm{S1}$ stimulation.

${ }^{b}$ Change (percentage) in the S1 fEPSP slope amplitude after $\$ 2$ stimulation.

concentration was no higher than $0.001 \% \mathrm{DMSO} / \mathrm{ACSF}$ ). To rule out any effect of these drugs on baseline stability, we performed recordings in the stratum oriens and the stratum radiatum and found that baseline field potentials were not affected by actinomycin-D, anisomycin, and KT5720 at the concentrations and incubation times used in our study (supplemental Table 4, available at www.jneurosci.org as supplemental material). In addition, our previous study (Barco et al., 2002) showed that VP16-cAMP response element-binding protein (CREB) expression did not affect the stability of baseline field potentials or basal synaptic transmission.

Statistical analysis. ANOVA analysis and Student's $t$ test were performed using the Origin statistical tool (Microcal Software Northampton, MA). For each set of drug-treated experiments, independent vehicle-control experiments were done. In the text, $n$ represents the number of animals. For single-pathway experiments, the number of slices tested (no more than two per animal) was also included in the statistical $n$. For matters of space and clarity, in some figures and tables, controls and vehicle conditions are shown as pooled data, but all statistical analyses are refereed to the corresponding control or vehicle condition. Data in the text and tables were presented as mean $\pm S D$, whereas in figures, they were presented as mean \pm SE (except in bar graphs, expressed as mean $\pm \mathrm{SD}$ ). The difference between the experimental data were considered significant at $p<0.05$.

\section{Results}

\section{Synaptic capture of L-LTP is similar in apical and}

\section{basal dendrites}

We recently reported the capture of L-LTP between two independent inputs within the stratum radiatum of the apical dendritic area of CA1 pyramidal neurons of the mouse hippocampus (Barco et al., 2002). Similarly to what was first described in rats (Frey and Morris, 1997, 1998a), a single $100 \mathrm{~Hz}$ train, which normally produces only the early phase of LTP (E-LTP), evoked L-LTP when L-LTP was previously induced by four trains at the other input (supplemental Fig. $3 B$, available at www.jneurosci.org as supplemental material) (for additional information, see Barco et al., 2002). In addition, we found that the expression of captured L-LTP was markedly sensitive to protein kinase A (PKA) activity and partially dependent on protein synthesis (see summary on Fig. 7D). To further characterize the process of synaptic capture, we now extend our study from the apical dendrites to the basilar dendrites.

Relatively few studies on L-LTP have been performed in basal dendrites (Otani et al., 1995; Son et al., 1996; Bradshaw et al., 2003). We therefore first characterized the general properties of 
L-LTP in basal dendrites in the stratum oriens of CA1 neurons and found that the properties of L-LTP were very similar in apical and basal dendrites. In both compartments, repeated trains of tetanic stimulation led to L-LTP (Fig. 1A,B). Moreover, L-LTP in basal and apical dendrites showed similar pharmacological properties, i.e., both were blocked by inhibitors of transcription and translation (Fig. $1 A, B$ ).

To test whether synaptic capture takes place in the basilar compartment, we performed two-pathway experiments within this compartment similar to those described for apical dendrites (Barco et al., 2002). We found that a single train of $100 \mathrm{~Hz}$ delivered to one pathway (S1) evoked E-LTP in that pathway without affecting the baseline in the second one (S2) (Fig. 2A). However, the induction of L-LTP by four trains of $100 \mathrm{~Hz}$ in one pathway within the basilar compartment was accompanied by a significant and transient modification of the baseline in the other pathway (Fig. $2 B$ ). The most likely explanation for the effect on the baseline of the S2 pathway is that the spatial restriction of the stratum oriens increases presynaptic interference between the two pathways (supplemental Fig. 1, available at www. jneurosci.org as supplemental material). However, we cannot rule out the existence of heterosynaptic forms of synaptic plasticity in basal dendrites that may account for this effect (Otani et al., 1995). Despite this limitation, we did observe capture of L-LTP within the basilar compartment (Fig. 2C: S1, 120-150 min mean amplitude, $151 \pm 10 \%$; S2, 25-40 min mean amplitude, $124 \pm$ $8 \%$; S2, 120-150 min mean amplitude, $148 \pm 16 \%)$. Additionally, we found that the expression of the capture of L-LTP in basal dendrites was sensitive to protein synthesis and PKA activity (Fig. 2D: S2 control, 120-150 min mean amplitude, $159 \pm 13 \%$; S2 anisomycin, 120-150 min mean amplitude, $132 \pm 14 \%$, $p=0.04$; S2 KT5720, 120-150 min mean amplitude, $126 \pm 12 \%$, $p=0.03)$. Our data on synaptic capture at basal dendrites corroborate those in apical dendrites (Barco et al., 2002) and support the hypotheses of Frey and Morris (1997) in the hippocampus and Martin et al. (1997) in Aplysia that newly made gene products
Basal dendrites

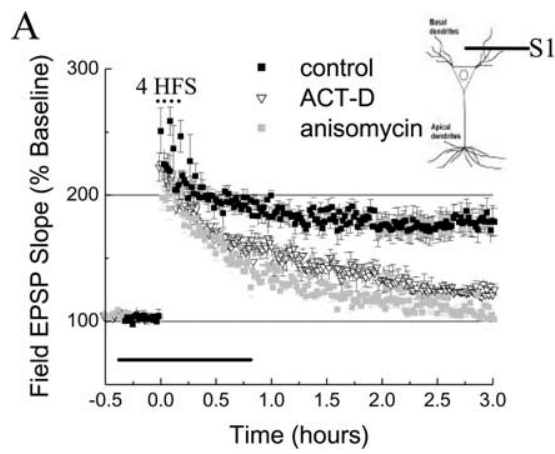

Figure 1. Late phase of LTP in basilar and apical dendritic compartments. A, L-LTP induced by four trains of $100 \mathrm{~Hz}$ (4 HFS) evoked at squares; $n=6$ ). $\boldsymbol{B}, \mathrm{L}$-LTP induced by four trains of $100 \mathrm{~Hz}$ evoked at apical dendrites (filled circles; $n=6$ ) is disrupted by actinomycin-D ( ACT-D, open triangles; $n=6$ ) and anisomycin (gray circles; $n=6$ ). Horizontal bars represent the time the drug was applied onto slices. S1 and S2 represent pathways synapsing on basal and apical dendrites of the CA1 area, respectively. Data are represented as mean \pm SEM.

are available to synapses other than those that triggered their synthesis.

\section{Synaptic tagging in basal and apical dendrites}

One single train of high-frequency stimulation is sufficient to trigger intracompartmental capture of L-LTP in both dendritic compartments, suggesting that synaptic tagging depends on similar signals in apical and basal dendrites. To further explore the mechanism of capture in both compartments, we investigated synaptic tagging and capture of L-LTP using two other paradigms. First, we used antidromic stimulation of CA1 pyramidal cell axons, which leads to the induction of gene expression and reduces the threshold for L-LTP so it can be achieved by a single train of stimulation (Dudek and Fields, 2002). Second, we investigated the LTP phenotype in basal and apical dendrites of VP16CREB transgenic mice. In this line of genetically modified mice, there is enhanced expression of CRE-driven genes and facilitated L-LTP (Barco et al., 2002). In both experimental models, nuclear activation and induction of CRE-driven gene expression can be achieved in the absence of previous synaptic activation and, consequently, tagging. Therefore, using these models, it was possible to dissect tagging from the induction of L-LTP. We found that, after antidromic stimulation of the pyramidal cells by four trains
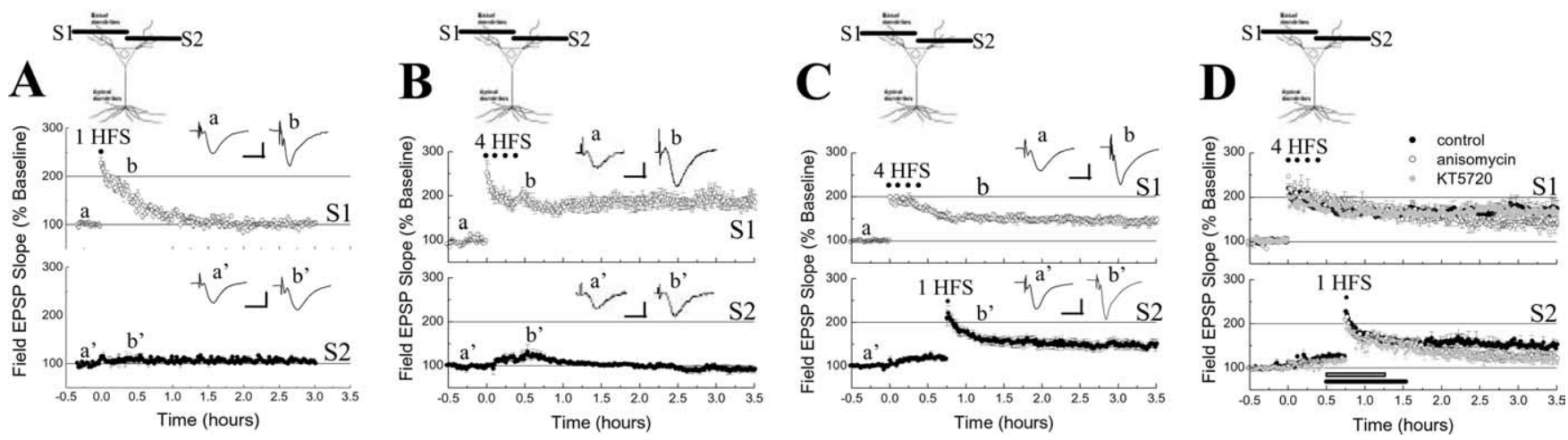

Figure 2. Basilar intracompartmental capture of L-LTP. A, E-LTP is induced by 1 train of $100 \mathrm{~Hz}$ (1 HFS) in basal dendrites (S1, open circles), whereas a second pathway (S2, filled circles) within the same dendritic compartment remains stable $(n=5)$. B, Four trains of $100 \mathrm{~Hz}$ (4HFS) induce L-LTP in basal dendrites (S1, open circles), but it also affects the stability of the baseline in the second pathway (S2, filled circles) $(n=5)$. C, Capture of L-LTP was nevertheless observed in basal dendrites $(n=11)$. $\boldsymbol{D}$, Capture of L-LTP in basal dendrites is affected by anisomycin (20 $\mu \mathrm{m}$, black horizontal bar, open circles; $n=5)$ and KT5720 (1 $\mu \mathrm{m}$, gray horizontal bar, gray circles; $n=5)$. S1 and S2 represent two independent inputs to the basal dendritic layer of the CA1 area. Calibration: $1 \mathrm{mV}, 10 \mathrm{~ms}$. Data are represented as mean \pm SEM. 

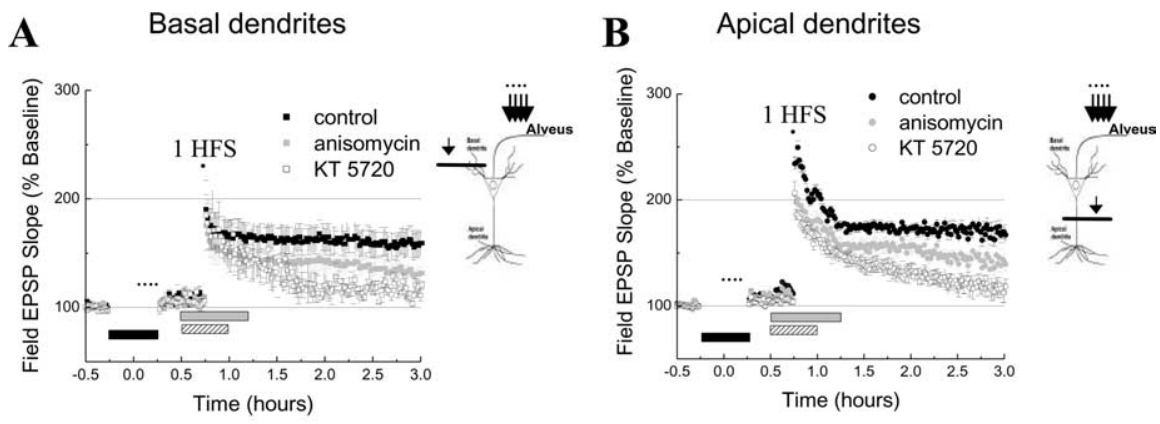

C
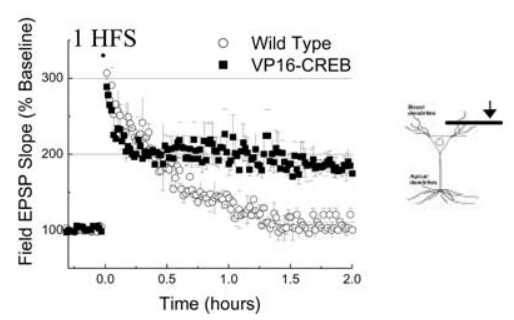

D

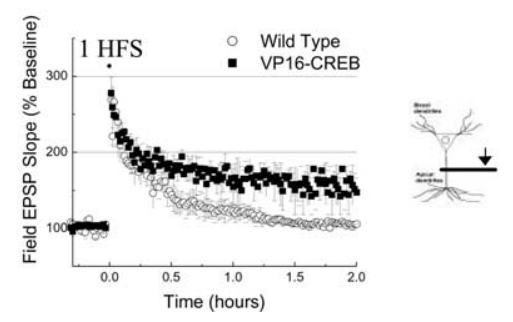

Figure 3. Synaptic tagging is similar in the apical and basilar dendritic compartments. $\boldsymbol{A}$, Antidromic stimulation facilitates the formation of L-LTP in basal dendrites. After antidromic stimulation (4 HFS; dots at $0 \mathrm{~h}$ ), one train of $100 \mathrm{~Hz}$ (1 HFS) delivered to basal dendrites induced L-LTP (filled squares; $n=6$ ). This form of L-LTP was disrupted by KT5720 ( $1 \mu \mathrm{m}$, dashed horizontal bar, open squares; $n=6$ ) and was reduced by anisomycin ( $20 \mu \mathrm{m}$, gray horizontal bar, gray squares; $n=6)$. $\boldsymbol{B}$, Antidromic stimulation also facilitated the formation of L-LTP in apical dendrites that was sensitive to KT5720 ( $1 \mu \mathrm{M}$, dashed horizontal bar, open circles; $n=6$ ) and anisomycin (20 $\mu \mathrm{m}$, gray horizontal bar, gray circles; $n=6$ ). In $\boldsymbol{A}$ and $\boldsymbol{B}$, kynurenic acid ( $3 \mathrm{~mm}$, black horizontal bar) was used to avoid synaptic activation from axon collaterals during antidromic stimulation. $C$, Facilitated L-LTP in basal dendrites by VP16-CREB expression (filled squares; $n=6$ ). $D$, Facilitated L-LTP in apical dendrites by VP16-CREB expression (filled squares; $n=6)$. Data are represented as mean \pm SEM.

A

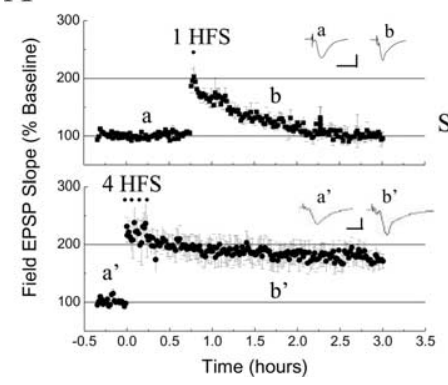

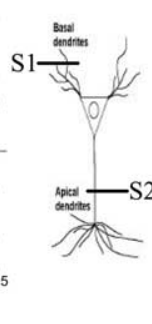

B

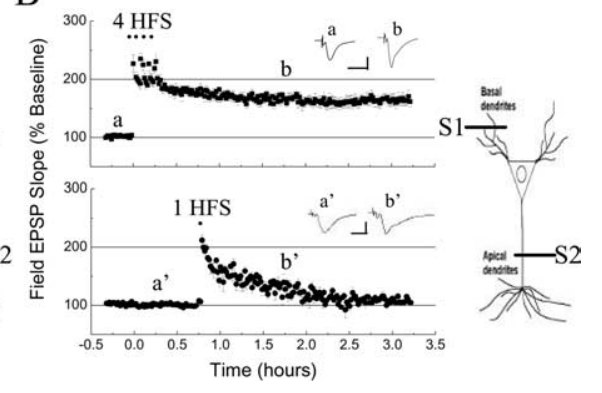

Figure 4. Failure to observe transcompartmental capture of L-LTP. $\boldsymbol{A}$, When L-LTP is evoked on apical dendrites (S2, filled circles), one train of $100 \mathrm{~Hz}$ (1 HFS) fails to elicit capture of L-LTP in basal dendrites (S1, filled squares; $n=6$ ). $\boldsymbol{B}$, When L-LTP is evoked in basal dendrites (S1, filled squares), one train of $100 \mathrm{~Hz}$ (1 HFS) fails to elicit capture of L-LTP in apical dendrites (S2, filled circles; $n=6$. Calibration: $1 \mathrm{mV}, 10 \mathrm{~ms}$. Data are represented as mean $\pm \mathrm{SEM}$.

of $100 \mathrm{~Hz}$ ( 5 min intertrain interval) delivered to the alveus, a single train of $100 \mathrm{~Hz}$ could induce capture of L-LTP in either basilar (Fig. 3A) or apical (Fig. 3B) dendritic compartments. Similarly, we found that, in VP16-CREB mice, one train of $100 \mathrm{~Hz}$ can successfully induce L-LTP in both basilar (Fig. 3C) and apical (Fig. 3D) dendritic compartments. Moreover, similar to the facilitated L-LTP in VP16-CREB mice (Barco et al., 2002), we found that both apical and basilar capture of L-LTP induced after antidromic stimulation are blocked by PKA inhibitors and reduced by inhibition of protein synthesis (Fig. $3 A, B$ ) (see Fig. 7D), two properties also observed in the case of intracompartmental synaptic capture (Barco et al., 2002). Because in these two protocols the marking signal circumvents synaptic-mediated nuclear activation, these results suggest that the same, or at least a very similar, mechanism may underlie synaptic tagging and capture in basilar and apical dendrites.

\section{A single train fails to capture L-LTP across dendritic compartments}

Because capture of L-LTP is possible within both the apical dendritic compartment and the basilar dendritic compartment. We next asked the following: does capture of L-LTP also occur across these two dendritic compartments? To perform these transcompartmental capture experiments, two independent synaptic inputs to the same neuronal population, located, respectively, in the stratum oriens and stratum radiatum, were stimulated using the same protocol described in our experiments in apical and basal dendrites. We found that, after the induction of L-LTP at apical dendrites, a single train of $100 \mathrm{~Hz}$, which would be sufficient to capture L-LTP in the same compartment, was not sufficient to capture L-LTP at basal dendrites (Fig. 4A: S1, 120-150 min mean basal amplitude, $101 \pm 10 \%$; S2, $120-150 \mathrm{~min}$ mean apical amplitude, $179 \pm 15 \%$ ). Similarly, when L-LTP was previously induced at basal dendrites, a single train of $100 \mathrm{~Hz}$ applied to apical dendrites was not sufficient either to evoke capture of L-LTP in apical dendrites (Fig. 4B: S1, 120-150 min mean basal amplitude, $167 \pm 11 \%$; S2, 120-150 min mean apical amplitude, $108 \pm 9 \%$ ).

Dendritic compartment-specific tagging The failure to induce capture of L-LTP across dendritic compartments with the same electrical stimulus (one train at 100 $\mathrm{Hz}$ ) that successfully induced capture of L-LTP within compartments suggests that synaptic tagging is compartment specific and that capture across compartments requires additional components than the activation of a synaptic tag. We therefore explored whether other parameters that may affect synaptic capture, such as (1) the intensity of the priming stimuli, (2) the time interval between the induction of L-LTP and the induction of capture of L-LTP, or (3) the distance between the nucleus and the site for L-LTP capture, might influence transcompartmental capture.

First, increasing the intensity of the priming signal at the site of initial marking from four to eight trains of $100 \mathrm{~Hz}$ in either apical or basilar dendrites did not promote the capture of L-LTP in the second compartment (Fig. 5A: S1, 180-210 min mean basal amplitude, $171 \pm 12 \%$; S2, 180-210 min mean apical amplitude, $117 \pm 12 \%$; and data not shown).

Second, decreasing or increasing the time interval for delivery of the gene products from $45 \mathrm{~min}$ to 15 or $90 \mathrm{~min}$, respectively, also failed to produce capture of L-LTP across dendritic compartments (Fig. 5B: 15 min interval, S2, 150-180 min mean apical 
A

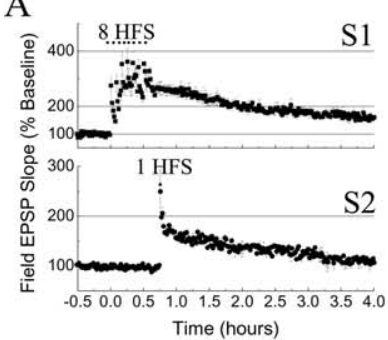

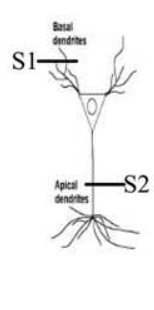

B

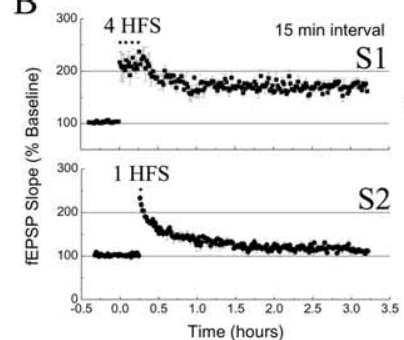

$\mathrm{C}$

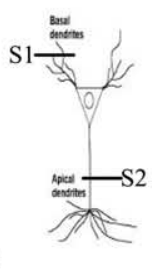

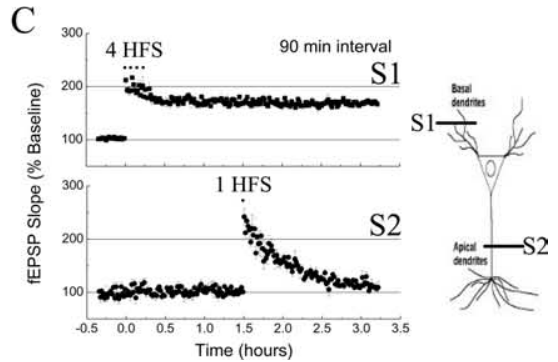

Figure 5. Failure to observe capture of L-LTP across dendritic compartments. $A$, An increase in the number of $100 \mathrm{~Hz}$ trains $(8 \mathrm{HFS})$ delivered to basal dendrites (S1, filled squares) did not facilitate capture of L-LTP in apical dendrites ( $S 2$, filled circles; $n=6$ ). $B$, One train of $100 \mathrm{~Hz}$ ( $1 \mathrm{HFS}$ ) fails to elicit capture of L-LTP in apical dendrites (S2, filled circles) 15 min after L-LTP is evoked on basal dendrites (S1, filled squares; $n=6$ ). C, One train of $100 \mathrm{~Hz}$ (1 HFS) fails to elicit capture of L-LTP in apical dendrites (S2, filled circles) 90 min after L-LTP is evoked in basal dendrites (S1, filled squares; $n=6) . S 1$ and $S 2$ represent independent afferents synapsing on the basal and the apical dendritic compartment, respectively. Data are represented as mean \pm SEM.

amplitude, $117 \pm 10 \%$; Fig. 5C: $90 \mathrm{~min}$ interval, S2, 150-180 min mean apical amplitude, $118 \pm 14 \%)$. These results indicate that the failure was not dependent on the time interval between stimuli delivered to each dendritic compartment.

Third, varying the distance between the capture site and the nucleus did not promote capture of L-LTP across dendritic compartments. Transcompartmental capture of L-LTP equally failed when tested at either the proximal or the distal stratum radiatum dendrites after L-LTP was originally induced at the basilar dendrites (supplemental Fig. 2, available at www. jneurosci.org as supplemental material). Moreover, apical intracompartmental capture of L-LTP was not enhanced when the capture site was closer to the nucleus, and we still observed capture of L-LTP within this compartment when the spatial separation between the two sites was similar than that used in transcompartmental capture (supplemental Fig. 3, available at www.jneurosci.org as supplemental material).

Finally, we considered that the failure for capturing L-LTP across compartments could be attributable to a restricted (i.e., compartment specific) utilization of gene products. Perhaps the induction of inputspecific L-LTP by repeated tetanic stimulation at either the stratum oriens or at the stratum radiatum promotes the distribution of gene products primarily in the same dendritic compartment in which this priming signal was given. Consequently, the utilization of gene products in a different compartment may need a stronger tagging signal.

In the attempt to assess different tagging signals, we used two trains of $100 \mathrm{~Hz}$ (30 s intertrain interval). This stimulation elicits an intermediate phase of LTP (I-LTP) (Winder et al., 1998) in both basal and apical dendrites, with transient levels of potentiation that lasts up to $3 \mathrm{~h}$ (Fig. $6 A, B$ ). This form of I-LTP exhibited, in both dendritic compartments, a strong requirement for PKA activity, a rather modest requirement for protein synthesis, and no requirement for transcription (Winder et al., 1998; Woo and Nguyen, 2003) (Fig. 6A,B) (supplemental Table 1, available at

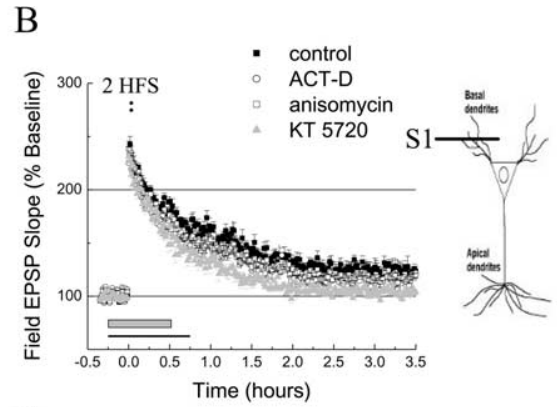

$\mathrm{D}$

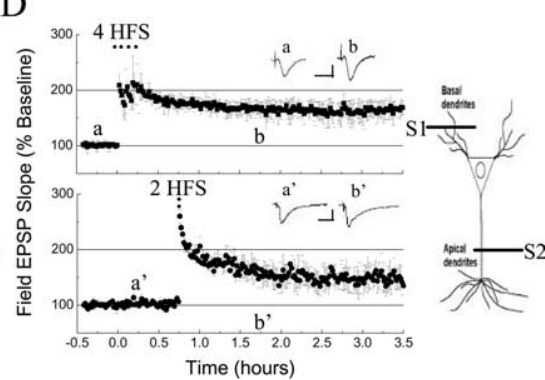

Figure 6. Transcompartmental capture of L-LTP elicited by I-LTP-inducing stimuli. A, I-LTP induced by two trains ( 30 s intertrain interval) of $100 \mathrm{~Hz}$ (2 HFS) in apical dendrites (filled circles; $n=6)$ is sensitive to KT5720 (1 $\mu \mathrm{m}$, gray horizontal bar, gray triangles), slightly affected by anisomycin ( $20 \mu \mathrm{m}$, black line, open circles; $n=6$ ) and not at all affected by actinomycin-D (ACT-D, , $\mu$, black line, open squares; $n=6$ ). $B$, I-LTP (evoked by 2 trains of $100 \mathrm{~Hz}, 30$ s intertrain interval; $2 \mathrm{HFS}$ ) is also observed in basal dendrites (filled squares), and it is only sensitive to KT5720 (1 $\mu \mathrm{m}$, gray horizontal bar, gray triangles; $n=5)$ but not to cin ( $20 \mu \mathrm{M}$, blackline, open squares; $n=5)$ or actinomycin-D ( $40 \mu \mathrm{M}$, black line, open circles; $n=5)$. C, Capture of L-LTP squares; $n=9$ ). $\boldsymbol{D}$, Capture of L-LTP across dendritic compartments can be elicited by two trains of $100 \mathrm{~Hz}$ ( 30 s intertrain interval; $2 \mathrm{HFS}$ ) in apical dendrites ( $S 2$, filled circles; $n=9$ ). Calibration: $1 \mathrm{mV}, 10 \mathrm{~ms}$. Data are represented as mean \pm SEM.

www.jneurosci.org as supplemental material) (but see Tsokas et al., 2005). We tested the ability of I-LTP-inducing stimuli to capture the late phase of LTP across compartments and found that two trains of $100 \mathrm{~Hz}$ delivered to afferents fibers on basal dendrites after the induction of L-LTP at apical dendrites successfully captured a late-phase LTP lasting $>3 \mathrm{~h}$ (Fig. 6C). Similar results were obtained when we evoked L-LTP at basal dendrites and applied I-LTP-inducing stimuli to afferents fibers on apical dendrites (Fig. $6 D$ ). These data indicate that the capture of L-LTP can indeed occur across dendritic compartments, but it requires a stronger tagging stimulus than intracompartmental capture of L-LTP (supplemental Fig. 4, available at www.jneurosci.org as supplemental material).

We then investigated some biochemical properties of the 
A

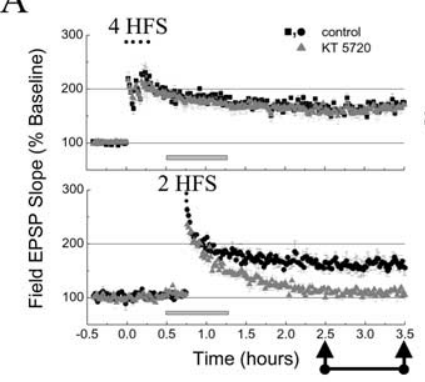

$\mathrm{C}$
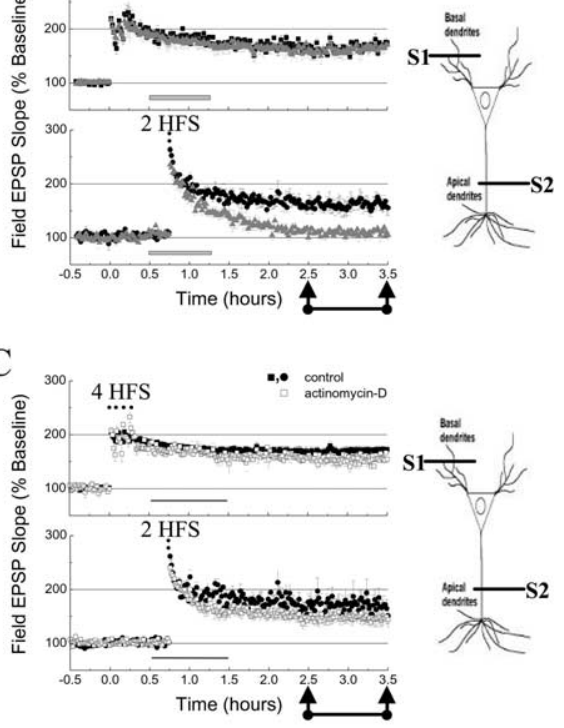

B
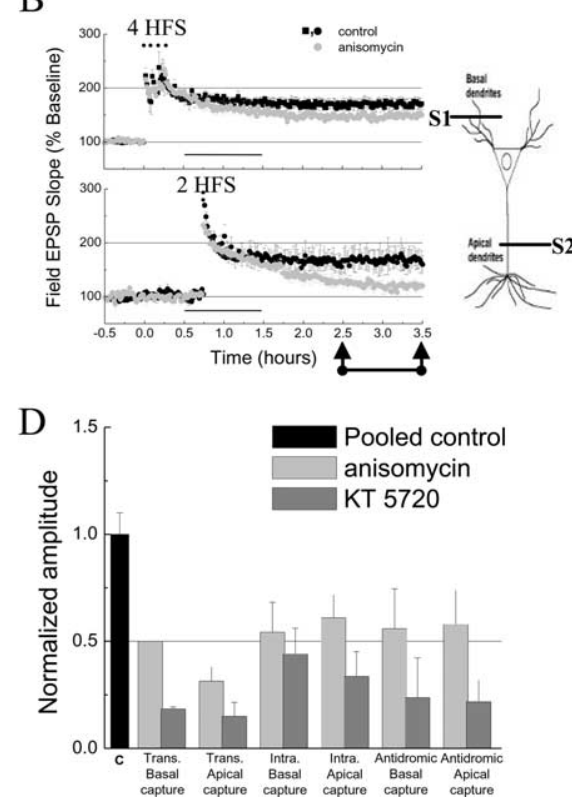

Figure 7. Pharmacological characterization of the transcompartmental capture of L-LTP. Sensitivity to KT5720 $(1 \mu \mathrm{M} ; \boldsymbol{A})$, anisomycin $(20 \mu \mathrm{m} ; \boldsymbol{B})$, and actinomycin-D (40 $\mu \mathrm{m} ; \boldsymbol{C}$ ) of transcompartmental capture of L-LTP in apical dendrites (S2). The gray (thick) and black (thin) horizontal bars represent the time of KT5720, anisomycin, and actinomycin-D application onto slices. $n=$ 6 for each independent data set. $\boldsymbol{D}$, Histogram represents the normalized amplitude of captured L-LTP in the absence (black bar) and in the presence of translation (light gray bars) and PKA inhibitors (dark gray bars) observed in different experimental paradigms (for apical intracompartmental capture: 150-210 min, control, $168 \pm 12 \%$; KT5720, $123 \pm 9 \%$; anisomycin, $141 \pm$ $11 \% ; n=5)$. Trans, Transcompartmental; Intra, intracompartmental. Data are represented as mean \pm SEM.

mechanism for transcompartmental capture of L-LTP at the apical dendritic compartment and found that, as in the case of intracompartmental capture, it was sensitive to PKA activation (Fig. 7A, S2, gray triangles), partially dependent on protein synthesis (Fig. $7 B, S 2$, gray circles), and not significantly affected by transcription inhibition (Fig. 7C, S2, gray squares) (for values, see supplemental Table 2, available at www.jneurosci.org as supplemental material). Similar results were obtained when we assayed transcompartmental capture of L-LTP in the basilar dendritic compartment and L-LTP was induced in the apical dendritic compartment (supplemental Table 3, available at www. jneurosci.org as supplemental material). Figure $7 D$ summarizes these findings and highlights the inhibitory effects of KT5720 and anisomycin in the different experimental conditions used in our study. Transcompartmental capture, therefore, appears to share the same molecular mechanisms, dependence on PKA activity, and protein synthesis, as intracompartmental capture (Barco et al., 2002).

\section{Discussion \\ Synaptic tags, synaptic capture, and dendritic compartment-specific signals}

The term synaptic capture describes the ability of gene products (mRNAs and proteins) newly synthesized in the cell body to be used productively by those synapses marked with an activitydependent tag (Frey and Morris, 1997, 1998b; Martin et al., 1997). A number of studies in Aplysia and in the stratum radiatum of the hippocampus support this model and identified some of the molecular events involved in this process (Barco et al., 2002, 2005; Dudek and Fields, 2002; Martin and Kosik, 2002). They show that it relies on four critical activity-mediated cellular mechanisms: (1) synaptic marking, (2) nuclear activation, (3) translation activation, and (4) synaptic capture of new gene products. Here we extended previous studies on synaptic capture in two ways: (1) we investigated the interaction between two inputs within the stratum oriens, and (2) we explored the interaction across the apical and basilar dendritic compartments of CA1 neurons.

We found that, despite the differences in the mechanisms for induction and expression of LTP in basal and apical dendrites (Arai et al., 1994; Leung and Shen, 1995; Otani et al., 1995; Haley et al., 1996; Son et al., 1996; Cavus and Teyler, 1998; Kloosterman et al., 2001; Kawakami et al., 2003; Kramar and Lynch, 2003), E-LTP, L-LTP, and intracompartmental capture of L-LTP in each dendritic compartment could be induced with the same kind of stimulation protocols. In addition, we observed that the capture of L-LTP across dendritic compartments required a stronger marking stimulation than that required for the capture of L-LTP within the same compartment.

One possible explanation for this difference is that these two forms of capture require different types of synaptic tags. $\mathrm{Al}$ though this option cannot be ruled out, three independent lines of evidence suggest that, in the apical and basilar dendritic compartments, tagging and capture share the same mechanisms: (1) intracompartmental capture of L-LTP was similarly elicited in both dendritic areas; (2) experiments using strong antidromic stimulation of CA1 pyramidal cells (Dudek and Fields, 2002) showed that E-LTP-inducing stimuli can successfully capture L-LTP in either the basilar or the apical dendritic compartment; and (3) experiments with VP16-CREB mice, in which CRE-driven genes are already expressed, provided similar results and led to the same conclusions as antidromic stimulation. Together, these data indicate that $100 \mathrm{~Hz}$ train is sufficient to set a synaptic tag within any compartment.

Synaptic tagging is, by definition, a local event restricted to the stimulated synapses and should not depend on how or where the synaptic activation that initiated gene induction took place. To explain the failure of one train to induce transcompartmental capture of L-LTP, we propose a model according to which the mark is compartment restricted (Fig. 8). This dendritic compartment-specific mark might contribute to enhance both the transport of new gene products to the stimulated dendritic compartment and their use [i.e., local mRNA translation (Sutton and Schuman, 2005)]. Interestingly, Fonseca et al. (2004) have demonstrated recently that the interaction between two synaptic inputs expressing L-LTP is also restricted to a given dendritic compartment (Fonseca et al., 2004).

We found that an intermediate stimulation, two trains of 100 $\mathrm{Hz}$, appears to be required to mark the compartment as suitable for transcompartmental capture of L-LTP by setting tags not only at the site of the activated synapses but also within that dendritic compartment. Perhaps synaptic capture depends on the activity level within a compartment, which could be reached by stimulation equal or larger than two trains of $100 \mathrm{~Hz}$ or after strong antidromic stimulation. It is also possible that there could be 
compartment-selective resources that are required for tags set by conventional one train at $100 \mathrm{~Hz}$ compared with two trains at $100 \mathrm{~Hz}$, or that the stronger tagging stimuli, which itself induces I-LTP, leads to a different kind a L-LTP during transcompartmental capture. Thus, our findings both restrict and extend the idea of synaptic tagging of Frey and Morris (1997, 1998b) to include the existence of an additional activity-mediated mechanism that we called compartment-specific marking.

\section{Capture of L-LTP in VP16-CREB mice} and after antidromic stimulation

Our experiments using VP16-CREB mice and antidromic stimulation show that new gene products could be captured in both basilar and apical dendritic compartments with the same stimulation (one train at $100 \mathrm{~Hz}$ ) as intracompartmental capture. Conceivably, constitutively active gene expression initiated in the absence of synaptic activity, as in the case of VP16-CREB mice, results in gene products evenly distributed within the cell by default; hence, only activation of the local synaptic tag (one train at $100 \mathrm{~Hz}$ ) is required for capture of L-LTP (Barco et al., 2002). Strong, nonsynaptic, antidromic stimulation of the cell body might also allow a homogeneous distribution of gene products into the cell (Dudek and Fields, 2002). We suggest that antidromic stimulation of the soma, in addition to activating gene expression (Dudek and Fields, 2001), leads to activation of the compartment tag in both basilar and apical dendrites. Stimulation of the soma via signals originated in one dendritic compartment (transcompartmental experiments) also activates gene expression, but it might be insufficient to activate the compartment tag in the opposite dendritic compartment.

The mechanistic dissociation between dendritic and antidromic somatic activation might rely on the nature of the initial stimulation, i.e., synaptic or nonsynaptic (Adams and Dudek, 2005; Lee et al., 2005). Direct stimulation of axons in the alveus would lead to antidromic stimulation of the soma of the CA1 neurons that would exceed the dendritic threshold for compartment specificity. We suggest that this antidromic stimulation might generate spike backpropagation into both dendritic compartments, therefore activating dendritic compartment tags in each one (Kloosterman et al., 2001). Conversely, the effect of dendritic spikes of synaptic origin on compartment tags would be restricted to the same somatodendritic area.

Interestingly, the properties of synaptic capture observed in the bifurcated sensory neurons of Aplysia (Martin et al., 1997) resemble those described for intracompartmental capture in hippocampal CA1 neurons. Likely because, unlike the dendrites of a CA1 neuron, both neuronal branches of the Aplysia sensory neuron are part of the same cellular compartment or because synaptic capture in Aplysia occurred in a cellular state different as that described in this study.
What is the molecular nature of the compartment-specific mark? Conceivably, the selective and activity-mediated tagging of cytoskeleton structures (Sanchez et al., 2000; Luo, 2002; Bradshaw et al., 2003) by PKA might enhance the transport of mRNAs and proteins to dendrites containing activated synapses (Kotz and McNiven, 1994; Rodionov et al., 2003). According to this view, the gene products induced by signals that reach the nucleus would be primarily distributed back into the same dendritic compartment in which the activation cascade was initiated, whereas little would be distributed to the other dendritic compartments that were not activated. In agreement with this view, Steward et al. (1998) found that Arc mRNA and protein accumulate selectively in previously activated dendritic domains of the molecular layer at the dentate gyrus. When Arc mRNA was induced by electroconvulsive seizure instead of by specific stimulation of entorhinal cortical projections, it was distributed homogeneously throughout the molecular layer. These different patterns of Arc mRNA distribution suggest that, after synaptic activation, the mRNA molecules entering the dendrites are actively redistributed to the activated zone and depleted from nonactivated regions (Steward et al., 1998). Similarly, Guistetto et al. (2003) found that synaptic stimulation in Aplysia neurons strongly enhanced the transport of Ap-eEF1A mRNAs to the axon processes, whereas its transcripts tended to accumulate around the nucleus when the stimulation was restricted to the cell body.

In addition to the selective targeting of mRNA and protein, restricted or localized $\mathrm{mRNA}$ translation might potentially be enhanced by compartment-specific marking as suggested by the increased dependence on protein synthesis of transcom- 
partmental capture compared with intracompartmental capture. This view is also supported by studies showing that input-specific stimulation delivered to the apical dendrites causes localized mRNA translation in the same dendritic compartment (Ouyang et al., 1997, 1999; Steward and Halpain, 1999; Huang et al., 2005).

\section{Capture of L-LTP in structurally polarized cells}

In total, to achieve synapse specificity in cells with a complex polar architecture, mRNAs and proteins could travel from the soma to specific synapses in the cell (mail hypothesis), a possibility that requires the existence of very elaborate mechanisms of intracellular trafficking regulation. Conversely, gene products could be distributed cell wide to fulfill the requirement of a specific set of synapses (synaptic tag hypothesis). The existence of compartment-specific tags might significantly increase the efficacy of synaptic tags without involving unknown and complicated pathways of intracellular trafficking and would enhance the efficiency of mRNAs translation and protein capture that otherwise would be degraded without use. The differential requirement for transcompartmental and intracompartmental capture enhances the integrative capabilities of hippocampal neurons, enabling CA1 neurons to integrate differently the signals arriving from apical and basilar afferent inputs by setting different thresholds for integration.

\section{References}

Adams JP, Dudek SM (2005) Late-phase long-term potentiation: getting to the nucleus. Nat Rev Neurosci 6:737-743.

Amaral DG, Witter MP (1989) The three-dimensional organization of the hippocampal formation: a review of anatomical data. Neuroscience 31:571-591.

Arai A, Black J, Lynch G (1994) Origins of the variations in long-term potentiation between synapses in the basal versus apical dendrites of hippocampal neurons. Hippocampus 4:1-9.

Barco A, Alarcon JM, Kandel ER (2002) Expression of constitutively active CREB protein facilitates the late phase of long-term potentiation by enhancing synaptic capture. Cell 108:689-703.

Barco A, Patterson S, Alarcon JM, Gromova P, Mata-Roig M, Morozov A, Kandel ER (2005) Gene expression profiling of facilitated L-LTP in VP16-CREB mice reveals that BDNF is critical for the maintenance of LTP and its synaptic capture. Neuron 48:123-137.

Bradshaw KD, Emptage NJ, Bliss TV (2003) A role for dendritic protein synthesis in hippocampal late LTP. Eur J Neurosci 18:3150-3152.

Casadio A, Martin KC, Giustetto M, Zhu H, Chen M, Bartsch D, Bailey CH, Kandel ER (1999) A transient, neuron-wide form of CREB-mediated long-term facilitation can be stabilized at specific synapses by local protein synthesis. Cell 99:221-237.

Cavus I, Teyler TJ (1998) NMDA receptor-independent LTP in basal versus apical dendrites of CA1 pyramidal cells in rat hippocampal slice. Hippocampus 8:373-379.

Deuchars J, Thomson AM (1996) CAl pyramid-pyramid connections in rat hippocampus in vitro: dual intracellular recordings with biocytin filling. Neuroscience 74:1009-1018.

Dolleman-Van Der Weel MJ, Witter MP (1996) Projections from the nucleus reuniens thalami to the entorhinal cortex, hippocampal field CA1, and the subiculum in the rat arise from different populations of neurons. J Comp Neurol 364:637-650.

Dudek SM, Fields RD (2001) Mitogen-activated protein kinase/extracellular signal-regulated kinase activation in somatodendritic compartments: roles of action potentials, frequency, and mode of calcium entry. J Neurosci 21:RC122(1-5).

Dudek SM, Fields RD (2002) Somatic action potentials are sufficient for late-phase LTP-related cell signaling. Proc Natl Acad Sci USA 99:3962-3967.

Fonseca R, Nagerl UV, Morris RG, Bonhoeffer T (2004) Competing for memory: hippocampal LTP under regimes of reduced protein synthesis. Neuron 44:1011-1020.
Frey U, Morris RG (1997) Synaptic tagging and long-term potentiation. Nature 385:533-536.

Frey U, Morris RG (1998a) Weak before strong: dissociating synaptic tagging and plasticity-factor accounts of late-LTP. Neuropharmacology 37:545-552.

Frey U, Morris RG (1998b) Synaptic tagging: implications for late maintenance of hippocampal long-term potentiation. Trends Neurosci 21:181-188.

Giustetto M, Hegde AN, Si K, Casadio A, Inokuchi K, Pei W, Kandel ER, Schwartz JH (2003) Axonal transport of eukaryotic translation elongation factor lalpha mRNA couples transcription in the nucleus to longterm facilitation at the synapse. Proc Natl Acad Sci USA 100:13680-13685.

Haley JE, Schaible E, Pavlidis P, Murdock A, Madison DV (1996) Basal and apical synapses of CA1 pyramidal cells employ different LTP induction mechanisms. Learn Mem 3:289-295.

Huang F, Chotiner JK, Steward O (2005) The mRNA for elongation factor $1 \alpha$ is localized in dendrites and translated in response to treatments that induce long-term depression. J Neurosci 25:7199-7209.

Kandel ER (2001) The molecular biology of memory storage: a dialog between genes and synapses. Biosci Rep 21:565-611.

Katz B, Miledi R (1970) Further study of the role of calcium in synaptic transmission. J Physiol (Lond) 207:789-801.

Kawakami R, Shinohara Y, Kato Y, Sugiyama H, Shigemoto R, Ito I (2003) Asymmetrical allocation of NMDA receptor epsilon2 subunits in hippocampal circuitry. Science 300:990-994.

Kloosterman F, Peloquin P, Leung LS (2001) Apical and basal orthodromic population spikes in hippocampal CA1 in vivo show different origins and patterns of propagation. J Neurophysiol 86:2435-2444.

Kotz KJ, McNiven MA (1994) Intracellular calcium and cAMP regulate directional pigment movements in teleost erythrophores. J Cell Biol 124:463-474.

Kramar EA, Lynch G (2003) Developmental and regional differences in the consolidation of long-term potentiation. Neuroscience 118:387-398.

Lee PR, Cohen JE, Becker KG, Fields RD (2005) Gene expression in the conversion of early-phase to late-phase long-term potentiation. Ann NY Acad Sci 1048:259-271.

Leung LS, Shen B (1995) Long-term potentiation at the apical and basal dendritic synapses of CAl after local stimulation in behaving rats. J Neurophysiol 73:1938-1946.

Leung LS, Shen B (1999) N-methyl-D-aspartate receptor antagonists are less effective in blocking long-term potentiation at apical than basal dendrites in hippocampal CA1 of awake rats. Hippocampus 9:617-630.

Luo L (2002) Actin cytoskeleton regulation in neuronal morphogenesis and structural plasticity. Annu Rev Cell Dev Biol 18:601-635.

Martin KC (2002) Synaptic tagging during synapse-specific long-term facilitation of Aplysia sensory-motor neurons. Neurobiol Learn Mem 78:489-497.

Martin KC, Kosik KS (2002) Synaptic tagging-who's it? Nat Rev Neurosci 3:813-820.

Martin KC, Casadio A, Zhu H, Yaping E, Rose JC, Chen M, Bailey CH, Kandel ER (1997) Synapse-specific, long-term facilitation of Aplysia sensory to motor synapses: a function for local protein synthesis in memory storage. Cell 91:927-938.

Otani S, Connor JA, Levy WB (1995) Long-term potentiation and evidence for novel synaptic association in CA1 stratum oriens of rat hippocampus. Learn Mem 2:101-106.

Ouyang Y, Kantor D, Harris KM, Schuman EM, Kennedy MB (1997) Visualization of the distribution of autophosphorylated calcium/calmodulindependent protein kinase II after tetanic stimulation in the CA1 area of the hippocampus. J Neurosci 17:5416-5427.

Ouyang Y, Rosenstein A, Kreiman G, Schuman EM, Kennedy MB (1999) Tetanic stimulation leads to increased accumulation of $\mathrm{Ca}^{2+}$ / calmodulin-dependent protein kinase II via dendritic protein synthesis in hippocampal neurons. J Neurosci 19:7823-7833.

Pang PT, Lu B (2004) Regulation of late-phase LTP and long-term memory in normal and aging hippocampus: role of secreted proteins tPA and BDNF. Ageing Res Rev 3:407-430.

Pikkarainen M, Ronkko S, Savander V, Insausti R, Pitkanen A (1999) Projections from the lateral, basal, and accessory basal nuclei of the amygdala to the hippocampal formation in rat. J Comp Neurol 403:229-260. 
Rodionov V, Yi J, Kashina A, Oladipo A, Gross SP (2003) Switching between microtubule- and actin-based transport systems in melanophores is controlled by cAMP levels. Curr Biol 13:1837-1847.

Sanchez C, Diaz-Nido J, Avila J (2000) Phosphorylation of microtubuleassociated protein 2 (MAP2) and its relevance for the regulation of the neuronal cytoskeleton function. Prog Neurobiol 61:133-168.

Son H, Hawkins RD, Martin K, Kiebler M, Huang PL, Fishman MC, Kandel ER (1996) Long-term potentiation is reduced in mice that are doubly mutant in endothelial and neuronal nitric oxide synthase. Cell 87:1015-1023.

Steward O, Halpain S (1999) Lamina-specific synaptic activation causes domain-specific alterations in dendritic immunostaining for MAP2 and CAM kinase II. J Neurosci 19:7834-7845.

Steward O, Wallace CS, Lyford GL, Worley PF (1998) Synaptic activation causes the mRNA for the IEG Arc to localize selectively near activated postsynaptic sites on dendrites. Neuron 21:741-751.

Sutton MA, Schuman EM (2005) Local translational control in dendrites and its role in long-term synaptic plasticity. J Neurobiol 64:116-131.

Tsokas P, Grace EA, Chan P, Ma T, Sealfon SC, Iyengar R, Landau EM, Blitzer $\mathrm{RD}$ (2005) Local protein synthesis mediates a rapid increase in dendritic elongation factor 1A after induction of late long-term potentiation. J Neurosci 25:5833-5843.

Winder DG, Mansuy IM, Osman M, Moallem TM, Kandel ER (1998) Genetic and pharmacological evidence for a novel, intermediate phase of long-term potentiation suppressed by calcineurin. Cell 92:25-37.

Woo NH, Nguyen PV (2003) Protein synthesis is required for synaptic immunity to depotentiation. J Neurosci 23:1125-1132. 